\title{
Hypothesis Article: The Potential Therapeutic Effects of Colchicine for Prevention of Cytokine Storms Consequences in COVID-19 Patients
}

\author{
mohammad hablolvarid ${ }^{1}$ \\ ${ }^{1}$ Razi vaccine and serum research institute
}

July 7, 2020

\begin{abstract}
Background and Purpose. Since the onset of COVID-19 many clinical trials of drugs and/or vaccines are continuing and new ones are introduce daily. Yet the international research process failed to develop a preventative vaccine or potent therapeutic drugs for relieving the severity of the disease. Therefore, at present the best recommendation to people to slow the spread of the disease is; please stay at home and maintain social distancing! Experimental approach. Many aspects of pharmacotherapeutic mechanism of action of some previously approved drugs with anti-inflammatory effects, such as colchicine, in various disorders is not fully understood. Therefore, many potential therapeutic uses for these drugs and its analogues can be imagined. In this hypothesis article, an attempt has been made to evaluate some potential therapeutic effects of colchicine that may be benefit against COVID-19. Key Results. Prophylactic therapy with colchicine regulates the innate inflammatory response by blocking intracellular signaling pathways. This can inhibit respiratory alveolar destruction following COVID-19 pneumonia, which is the main cause of ARDS and death in these patients. Conclusion and Implications Since the macrophage and granulocytes are the main pro-inflammatory mediators in the lung, it seems application of therapeutic doses of colchicine, before the onset of respiratory problems, will protect the lung against severe damages and respiratory failure in COVID-19 patients. Obviously, many clinical trials are required to prove the validity of this claim.
\end{abstract}

\section{Conclusion and Implications}

Since the macrophage and granulocytes are the main pro-inflammatory mediators in the lung, it seems application of therapeutic doses of colchicine, before the onset of respiratory problems, will protect the lung against severe damages and respiratory failure in COVID-19 patients. Obviously, many clinical trials are required to prove the validity of this claim.

Before December 2019, no one in the world could have imagined that a novel severe pathogenic corona virus $(\mathrm{CoV})$ which later designated as severe acute respiratory syndrome coronavirus 2 (SARS-CoV-2) will soon cause a deep crisis in the whole world (Ren et al., 2020). In February 2020 the disease caused by this virus defined as coronavirus disease 2019 (COVID-19). Rapid spread of the disease in China followed by other countries caused the World Health Organization (WHO) in 11 Mar 2020 announced the disease a pandemic. Meanwhile, a consistent international work for development of potential vaccines and drugs with different mechanisms of action has been started. But, despite the fact that many clinical trials of COVID-19 drugs or vaccines are continuing and new ones are being added daily, scientists have been unable to find effective ways to slow the spread of the SARS-CoV-2 and treatment of COVID-19. Therefore, at present the best recommendation to people to slow the spread of the SARS-CoV-2 is, please stay at home and maintain social distancing!

Although most COVID-19 patients will develop mild to moderate symptoms of sickness, but, researchers argued that in minority of COVID-19 patients the oversecretion of pro-inflammatory cytokines and chemokines, 
often termed cytokine storm, play a crucial role in the fatal pneumonia observed after SARS-CoV-2 infections (Rahmati and mosavi., 2020; Zhang et al., 2020; Ruan et al., 2020). Different cytokine profiles in patients with severe COVID-19 have been reported but elevated levels of several cytokines/ chemokine such as interleukin 6 (IL-6), interleukin 10 (IL-10), interferon (IFN)-?, tumor necrosis factor (TNF) and Interferon gamma-inducible protein 10 (IP-10) have been greater emphasized in seriously ill patients, hospitalized in intensive care unit (ICU), than patient with mild to moderate symptoms ( Rahmati and Mosavi., 2020; Han et al., 2020; Li et al., 2020; Zhou et al., 2020; Liu et al, 2020; Marietta et al., 2020). However, some researcher reiterate that most cytokines measured in COVID-19 patients were not elevated above the upper limit of normal and few slightly above normal levels are indication of an infection related inflammatory response rather than hypercytokinemia (Liu et al., 2020). In other words these proinflammatory markers may be a consequence of the organ damage caused by the infection rather than the driver of acute respiratory distress syndrome (ARDS) caused by this infectious agent (Ruan et al., 2020). it is very important to understand that magnitude of the elevation of inflammatory cytokines in COVID-19 patient is truly a unique phenomenon or simply one that results in many similar conditions that cause severe damage to multiple organs such as in secondary haemophagocytic lymphohistiocytosis (sHLH), severe sepsis or in ARDS (Gu et al., 2019). Until now, the cytokine profile of COVID-19 patients with different disease severity is not clear (Han et al., 2020) and patients with severe COVID-19 represent different cytokine patterns. Therefore, multiple trials of immunosuppressive therapies has been used to test this hypothesis, so, more care should be taken before immunosuppressive therapy by cytokine blockers. Several approaches including total targeting of the inflammation or neutralizing a single key inflammatory mediator such as interleukin-6 (IL-6) are employed to manage cytokine storm in COVID-19 patients (Zhang et al., 2020). Though the results of a recent report provides further evidence that IL-6 in not elevated in COVID-19 patients. It should be emphasized that, computed tomography (CT) images taken from patients with SARS-CoV-2 infected pneumonia revealed characteristic white patches containing fluid in the lungs (Wang et al., 2020). The predominant histological pattern of lung injuries in 38 patients who died from COVID-19 in Italy was reported as: capillary congestion, interstitial and intraalveolar edema, dilated alveolar ducts and collapsed alveoli, hyaline membranes composed of serum proteins and condensed fibrin, and loss of pneumocytes. In fact following pulmonary injury, elevated levels of hyaluronan (HA) matrices accumulate within the airway submucosa, pulmonary vasculature walls, and, to a lesser extent, the alveoli and in respiratory secretions, correlating with the extent of injury (Lauer et al., 2015). A review of the literature reveals that HA has been associated with the pathophysiology of acute respiratory distress syndrome (ARDS). However, its key role in driving the morbidity and mortality of the condition has not yet been fully recognized (Hallgren et al., 1989). HA can absorb vast amounts of water, therefore, accumulation of this matrics in the pulmonary alveoli disrupt oxygen exchanges and leads the patient to ARDS (Hallgren et al., 1989; Shi et al., 2020; $\mathrm{Xu}$ et al., 2020). High level of inflammatory cytokines such as IL-1 and TNF was detected in the lung of COVID-19 infected patients. These cytokines have key role in induction of HA-synthase-2 (HAS2) in CD31+ endothelium cells; lung alveolar epithelial cell adhesion molecule positive (EPCAM+) cells and fibroblasts (Bell et al., 2020; Shi et al., 2020). It must be emphasized that there is much to be yet understood about various cytokines in the genesis of acute respiratory distress and pneumonia. However, every action for reduction or inhibition of production of HA, such as decrease IL-1 and TNF levels, is very useful for easier breathing of COVID-19 patients and suppressing the inflammation must be performed as the main efforts to manage the disease, hence it is wise to screen all patients with severe COVID-19 for hyperinflammation and determining the subgroups of patients for whom immunosuppression therapy may play a therapeutic role in combating disease and decreasing mortality. To quiet such pro-inflammatory factors production, researcher are now trying to find effective immunosuppressant therapeutic agents for COVID-19 patients. For instance, anakinra (human IL-1 receptor antagonist); tocilizumab (humanized monoclonal antibody against the IL-6 receptor) and Janus kinase (JAK) inhibitors that have therapeutic application in the treatment of inflammatory diseases such as rheumatoid arthritis, showed significant survival benefit in patients with hyperinflammation and some has been approved in patients with COVID-19 pneumonia (González-Gay et al., 2020) But, corticosteroids because of exacerbation of lung injury, are not recommended (Russel et al., 2020) It is important to remember that the pharmacotherapeutic mechanism of action of some previously 
approved drugs with proven anti-inflammatory effects, such as colchicine, in various disorders is not fully understood. Therefore, many potential therapeutic uses for colchicine and its analogues such as COVID-19 treatment could be expected. A Persian proverb says: water is in a jar and we are thirsty for lips!

Colchicine has been one of the first line therapy for the treatment of acute gouty arthritis and familial Mediterranean fever (FMF), but, due to the anti-inflammatory and anti-fibrotic activities, other therapeutic benefits of colchicine such as Behcet's disease (BD) has been determined (Leung et al., 2015). In the following paragraphs, some therapeutic effects of colchicine that are in consistent with this suggestion, are described:

Actually, colchicine down regulates multiple inflammatory pathways and modulates innate immunity. The main inhibitory mechanisms of actions of colchicine are inhibition of neutrophil chemotaxis, adhesion, mobilization and also blockage of superoxide production. Moreover, colchicine inhibit NACHT-LRRPYD-containing protein 3 (NALP3) inflammasomes activation and interleukin (IL) $1 \beta$ processing and release. Through microtubule depolymerisation, colchicine interferes with neutrophil adhesion and recruitment to inflamed tissue Furthermore, Colchicine has been shown to reduce oxidative stress by reducing calcium (Ca) influx into neutrophils (Leung et al., 2015). It has been shown that, even at nano concentrations (3 nanomolar) colchicine as a prophylactic drug can be used for elimination of adhesiveness of neutrophils to endothelial cells and at higher concentrations (300 nanomolar) it prevents further neutrophil recruitment (Cronstein et al., 1995). Moreover, at higher concentrations (5 micromolar), colchicine suppresses monosodium urate (MSU) induced NALP3 inflammasomes, responsible for caspase-1 activation and subsequent IL1 $\beta$ and IL18 processing and release (Martinon et al., 2006). Therefore, low prophylactic dose of colchicine could achieve a high enough intracellular concentration in macrophages to inhibit NALP3 inflammasome activation. Colchicine may also increase the threshold for initiation of complete NALP3 inflammasome activation to some extent by reducing subclinical inflammation (Pascual and Castellano., 1992). Prophylactic therapy with colchicine also regulates the innate inflammatory response by blocking intracellular signaling pathways by targeting nuclear factor $\mathrm{kB}$ (NF-kB) or caspase-1. Colchicine was also shown to modulate lipopolysaccharide-induced secretion of tumor necrosis factor (TNF) by liver macrophages in a rat model. Furthermore, colchicine is a potent inhibitor of pore formation induced by activation of both purinergic receptors P2X7 and P2X2 both in vitro and in vivo. The formation of P2X7 pores is a necessary step in the innate immune response for triggering ATP-induced NALP3 inflammasome activation (Leung et al., 2015; Marques-da-Silva et al., 2011)

In the cases of COVID-19 pneumonia, the epithelium of respiratory alveoli are destroyed and filled with fluid and inflammatory cells, so the lung unable to take on oxygen and get rid of carbon dioxide. Actually, this is the main cause of ARDS and death in these patients. Therefore, suppressing the inflammation must be performed as the main efforts to manage the disease. Since the macrophage and granulocytes are the main pro-inflammatory mediators in the lung, it seems application of therapeutic doses of colchicine, before the onset of respiratory problems, will protect the lung against severe damages and respiratory failure in COVID19 patients. Obviously, many clinical trials are required to prove the validity of this claim. However, narrow therapeutic index of colchicine with no clear cut off distinction between nontoxic, toxic, and lethal doses as well as unique route of, oral, administration are the main disadvantages of this drug (Finkelstein et al., 2010). Nevertheless, the benefits of intratracheally application of hyaluronidase for inhibition of hyaluronan synthase 2 (HAS2) enzyme and fluid accumulation in the lungs has already been recommended (Shi et al., 2020; Xu et al., 2020).

\section{Competing interests:}

The author declare no competing interest.

\section{Author information:}

Mohammad Hassan Hablolvarid, Department of Animal Pathology and Epidemiology, Razi vaccine and serum research institute, Agricultural Research, Education and Extension Organization (AREEO), KarajIran.

\section{Corresponding author:}


Dr. Mohammad Hassan Hablolvarid, Veterinary Pathologist, Department of Animal Pathology and Epidemiology, Razi vaccine and serum research institute, Agricultural Research, Education and Extension Organization (AREEO), Karaj-Iran.

hablolvarid@yahoo.com

h.hablolvarid@rvsri.ac.ir

1. Bell, T. J., Brand, O. J., Morgan, D. J, et al (2018). Defective lung function following influenza virus is due to prolonged, reversible hyaluronan synthesis. Matrix Biol . 80 , 14-28.

2. Cronstein, B. N., Molad, Y., Reibman, J., et al (1995). Colchicine alters the quantitative and qualitative display of selectins on endothelial cells and neutrophils. J Clin Invest .96 (2), 994-1002.

3. Finkelstein, T., Aks, S., Hutson, J. R., Juurlink, D. R, et al (2010). Colchicine poisoning: the dark side of an ancient drug. Clin Toxicol (Phila) . 48 (5), 407-14. doi: 10.3109/15563650.2010.495348.

4. González-Gay, M. A., Mayo, J., Castañeda, S., Cifrián, J. M and Hernández-Rodríguez, J (2020). Tocilizumab: from the rheumatology practice to the fight against COVID-19, a virus infection with multiple faces, Expert Opinion on Biological Therapy ,20 (7), 717-723 doi: 10.1080/14712598.2020.1770222

5. Gu, Y., Hsu, A. C., Pang, Z., et al (2019). Role of the innate cytokine storm induced by the influenza A virus. Viral Immunol .32 (6), 244-251. doi:10.1089/ vim.2019.0032.

6. Hallgren, R., Samuelsson, T., Laurent, T.C., and Modig, J (1989). Accumulation of hyaluronan (hyaluronic acid) in the lung in adult respiratory distress syndrome. Am Rev Respir Dis . 139 , 682-7. doi: org/10.1164/ajrccm/139.3.682

7. Han, H., Ma, Q., Li, C., Liu, R., et al (2020). Profiling serum cytokines in COVID-19 patients reveals IL-6 and IL-10 are disease severity predictors. Emerging Microbes \& Infections ,9 (1), 1123-1130. doi: $10.1080 / 22221751.2020 .1770129$

8. Lauer, M. E., Dweik, R. A., Garantziotis, S., and Aronica, M. A (2015). The rise and fall of hyaluronan in respiratory diseases. Int J Cell Biol . 2015: 712507. doi: org/10.1155/2015/712507

9. Leung, Y. Y., Hui, L. L. Y., and Kraus, V. B (2015). Colchicine - update on mechanisms of action and therapeutic uses.Semin Arthritis Rheum . 45 (3), 341-350. doi:10.1016/j.semarthrit.2015.06.013.

10. Li, B., Feng, F., Yang, G., Liu, A., et al (2020). Immunoglobulin G/M and Cytokines Detections in Continuous Sera from Patients with Novel Coronaviruses (2019-nCoV) Infection. Available at: SS$\mathrm{RN}$ : https://ssrn.com/abstract=3543609 or http://dx.doi.org/10.2139/ssrn.3543609

11. Liu, Y., Zhang, C., Huang, F., Yang, Y., Wang, F., et al (2020). Elevated plasma level of selective cytokines in COVID-19 patients reflect viral load and lung injury. National Science Review . 7 (6), 1003-1011, doi.org/10.1093/nsr/nwaa037

12. Marietta, M., Ageno, W., Artoni, A., De Candia, E., et al (2020). COVID-19 and haemostasis: a position paper from Italian Society on Thrombosis and Haemostasis (SISET). Blood Transfusion = Trasfusione del Sangue. 18 (3), 167-169. doi: 10.2450/2020.0083-20

13. Marques-da-Silva, C., Chaves, M. M., Castro, N. G., et al (2011). Colchicine inhibits cationic dye uptake induced by ATP in P2X2 and P2X7 receptor-expressing cells: implications for its therapeutic action. Br J Pharmacol . 163 (5), 912-926.

14. Martinon, F., Pétrilli, V., Mayor, A., et al (2006). Gout-associated uric acid crystals activate the NALP3 inflammasome. Nature .440 (7081), 237-241.

15. Pascual, E., and Castellano, J. A (1992). Treatment with colchicine decreases white cell counts in synovial fluid of asymptomatic knees that contain monosodium urate crystals. J Rheumatol .19, 600603.

16. Rahmati, M., and Moosavi, MA (2020). Cytokine-targeted therapy in severely ill COVID-19 patients: Options and Cautions. EJMO .4 (2): 179-181. doi: 10.14744/ejmo.2020.72142

17. Ren, L. L., Wang, Y. M., Wu, Z. Q., et al (2020). Identification of a novel coronavirus causing severe pneumonia in human: a descriptive study. Chin Med J . 133 (9), 1015-1024. doi:10.1097/CM9.0000000000000722

18. Ruan, Q., Yang, K., Wang, W., Jiang, L., Song, J (2020). Clinical predictors of mortality due to COVID-19 based on an analysis of data of 150 patients from Wuhan, China. Intensive Care Med .46 
(5), 846-848. doi: 10.1007/s00134-020-05991-x.

19. Russell, C. D., Millar, J. E., and Baillie, J. K (2020). Clinical evidence does not support corticosteroid treatment for 2019-nCoV lung injury. The Lancet . 395 (10223), 473-75. doi: 10.1016/S01406736(20)30317-2.

20. Shi, Y., Wang, Y., Shao, C., et al (2020). COVID-19 infection: the perspectives on immune responses. Cell Death \&3 Differentiation . 27 , 1451-1454. doi:org/10.1038/s41418-020-0530-3

21. Wang, D., Hu, B., Hu, C., Zhu, F., Liu, X., Zhang, J., et al (2020). Clinical characteristics of 138 hospitalized patients with 2019 novel coronavirus-infected pneumonia in Wuhan, China. JAMA. [Epub ahead of print]. doi: org/10.1001/jama.2020.1585.

22. Xu, Z., Shi, L., Wang, Y., et al (2020). Pathological findings of COVID-19 associated with acute respiratory distress syndrome.Lancet Respir Med . 8 (4), 420-422. doi: 10.1016/S2213-2600(20)30076X

23. Zhang, C., Wu, Z., Li, J. W., Zhao, H., Wang, G. Q (2020). The cytokine release syndrome (CRS) of severe COVID-19 and Interleukin-6 receptor (IL-6R) antagonist Tocilizumab may be the key to reduce the mortality. International Journal of Antimicrobial Agents .55 (5), 05954 doi: 10.1016/j.ijantimicag.2020.105954

24. Zhou, Y., Fu, B., Zheng, X., Wang, D., Zhao, C., Sun, R., et al (2020). Pathogenic T cells and inflammatory monocytes incite inflammatory storm in severe COVID-19 patients. National Science Review . 7 (6), 998-1002, doi.org/10.1093/nsr/nwaa041 